\title{
A new approach to the estimation of surface free energy based on Vickers microhardness data
}

\author{
M. Staneva*, E. Nedkov \\ Institute of Polymers, Bulgarian Academy of Sciences, Acad. G. Bonchev Street, bl. 103A, Sofia 1113, Bulgaria
}

Received 4 December 2008; accepted in revised form 24 January 2009

\begin{abstract}
A relation between surface free energy $\left(\sigma^{M H V}\right)$ and Meyer's lines cut-values has been established using Vickers microhardness (MHV) method and empirical physical laws. This relation allows the calculation of $\sigma^{M H V}$ only from MHV data. The parameters required are Meyer's lines cut-values and the mean value of diagonal length of the impression at different loads applied $\left(d_{\text {real }}^{\text {mean }}\right)$. Our study of 12 samples of ultra high molecular weight polyethylene (PE-UHMW) showed that the new approach is applicable when the slope value of Meyer's lines equals 2 (i.e. $n=2$ ). A $\gamma^{-60}$ Co source was used for the irradiation of 11 samples (one of the samples investigated is un-irradiated) at room temperature in air. Doses of 1, 2, $5,10,20,50,100,200,500,1000$ and $1500 \mathrm{kGy}$ were applied. The values of $\sigma^{M H V}$ obtained are in a good agreement with the literature. The dependence of $\sigma^{M H V}$ on the dose applied strictly corresponds to the radiation effects theory. MHV was measured at seven different loads $-0.0123,0.0245,0.049,0.098,0.196,0.392,0.785 \mathrm{~N}$ at a loading time of $30 \mathrm{~s}$.
\end{abstract}

Keywords: material testing, Vickers microhardness (MHV), surface free energy

\section{Introduction}

\subsection{Fundamentals of Vickers microhardness (MHV) method}

The microhardness of a polymeric material - resistance to local deformation - is a complex property related to mechanical properties such as modulus, strength, elasticity and plasticity [1].

MHV test uses a square diamond pyramid, with angles $\alpha$ between non-adjacent faces of the pyramid of $136^{\circ}$ ([1], p. 3). The microhardness is estimated by Equation (1):

$$
M H V=\frac{2 \cdot P \cdot \sin \left(\frac{\alpha}{2}\right)}{d^{2}}=1.8544 \cdot \frac{P}{d^{2}}
$$

where $P$ is the force (the load applied), [N]; $d$ is the mean diagonal length of the impression after removing the indenter (pyramid), [mm]; $\mathrm{MHV}$ - in [MPa].

\subsection{Fundamentals of the approach - description of deriving the equation for surface free energy $\left(\sigma^{\mathrm{MHV}}\right)$}

Our new approach to estimating $\sigma^{M H V}$ is based on Meyer's power law represented by Meyer's line [2], as given by Equation (2a):

$\log P=\log a+n \cdot \log d$

where $a$ and $n$ are physical parameters, corresponding to elastic (strength) and plastic properties of the material [2,3]. A critical value for $n$ is observed $(n=2)$. At this value MHV is almost constant in depth. At $n<2$ or $n>2$, MHV is variable. It decreases $(n<2)$ or increases $(n>2)$, in the sample

*Corresponding author, e-mail: mstaneva@gmail.com

(C) BME-PT 
depth. This phenomenon has to be attributed to changes in the structure of the material $[2,4]$.

The dependence (2a) results in a straight line, where $\log a$ is the cut and $n$ is the slope. Parameter $a$ is determined using the antilogarithm of the cutvalue: $a=10^{\log a}$. Further on $a$ is denoted as 'cut resultant parameter' (CRP).

CRP (a) corresponds to the elastic (strength) properties of the material and its physical meaning has not been elucidated fully yet [2-4].

The aim of this work was to calculate $\sigma^{M H V}$ via CRP values applying empirical physical laws. Another form of Meyer's power law (Equation (2a)) is presented in Equation (2b):

$P=a \cdot d^{n}$

The CRP value ( $a$ ) can be calculated from Equation (1) and Equation (2b) (Equation (3)):

$$
a=\frac{M H V}{1.8544}=\frac{P}{d^{2}}
$$

Equation (3) is valid only when $n=2$.

Our new approach to estimating of $\sigma^{M H V}$ is based on the general requirement $n=2$ and applies empirical physical laws.

The following nine steps were used to obtain the final result.

\subsubsection{Evaluation of the experimental data viewing the fulfillment of the general requirement}

Setting $n=2$ as a general requirement is based on two considerations:

- The requirement $n=2$ results from Equation (3), which was used for deriving the relation between $\sigma^{M H V}, \mathrm{CRP}(a)$ and $d$ (see point (1.2.3) and (1.2.5) below).

- In the case $n \neq 2$, the unit of CRP $(a)$ is unknown and has no physical meaning:

- for $n<2$ (i. e. $n=1$ ), CRP (a) is measured in $\mathrm{N} / \mathrm{m}$;

- for $n>2$ (i. e. $n=3$ ), CRP $(a)$ is measured in $\mathrm{N} / \mathrm{m}^{3}$

In case $n=2$ the unit for CRP (a) is well defined and its physical meaning is a force applied to a unit area, $\left[\mathrm{N} / \mathrm{m}^{2}\right]$.

\subsubsection{Evaluation of the dimension of $d,[\mu \mathrm{m}]$}

As already mentioned Meyer's power law (Equation (2a)) is represented by a Meyer's line. For each straight line cut and slope can be calculated. The slope $(n)$ has to be 2 (see point 1.2.1). The cut $(\log a)$ is calculated every time when $\log d=0$, i.e. $d=1$. As in this case $d=1$, if the dimension of $d$ is $\mu \mathrm{m}$, than the mean diagonal length of the impression is $d=1 \mu \mathrm{m}$. That is why a very small microscopic impression $(d=1 \mu \mathrm{m})$ has been used in our study. Further on the diagonal length of this very small microscopic impression is denoted as $d_{1 \mu \mathrm{m}}$.

\subsubsection{Surface parameter $M H V^{*}$}

$M H V^{*}$ was derived using Equation (3) (Equation (4)):

$M H V^{*}=1.8544 \cdot 10^{12} \cdot a ;[\mathrm{Pa}]=\left[\frac{\mathrm{N}}{\mathrm{m}^{2}}\right]$

$M H V^{*}$ in Equation (4) is denoted as a surface parameter due to its unit $\left[\mathrm{N} / \mathrm{m}^{2}\right]$ corresponding to surface stress.

\subsubsection{Area of the very small microscopic impression with $d_{1} \mu \mathrm{m}\left(S_{1} \mu \mathrm{m}\right)$}

The equation given below was used for calculating $S_{1 \mu \mathrm{m}}$ (Equation (5)):

$S_{1 \mu \mathrm{m}}=\frac{2 \cdot 0.38 \cdot d_{1 \mu \mathrm{m}}^{2}}{\sqrt{2}} ; \quad\left[\mathrm{m}^{2}\right]$

Equation (5) was deduced after simple geometrical calculations. As the indenter is a diamond pyramid, the impression exhibits a pyramidal shape. The impression area is represented by the sum of the areas of the four pyramid walls. $d_{1 \mu \mathrm{m}}$ could be found on the top of the pyramid (impression).

\subsubsection{Force required for the formation of a very small microscopic impression (F)}

Multiplying the final results from point 1.2.3 $\left(M H V^{*}\right)$ and point 1.2.4 $\left(S_{1 \mu \mathrm{m}}\right)$ the Equation (6) is obtained: 


$$
M H V^{*} \cdot S_{1 \mu \mathrm{m}}=F ;\left[\frac{\mathrm{N}}{\mathrm{m}^{2}}\right]=\left[\mathrm{m}^{2}\right]
$$

\subsubsection{Calculation of mean values $\left(d_{\text {real }}^{\text {mean }}, h_{\text {real }}^{\text {mean }}\right)$}

For each sample a mean $d$ value for all loads applied is required to be calculated and is denoted as $d_{\text {real }}^{\text {mean }}$.

According to the geometry of the Vickers pyramid a relation between indentation depth $(h)$ and $d$ is derived (Equation (7)):

$$
h=\frac{d}{7} ;[\mathrm{m}]
$$

Using $d_{\text {real }}^{\text {mean }}$ and Equation (7) the mean $h$ value for all loads applied $\left(h_{\text {real }}^{\text {mean }}\right)$ for each sample is calculated.

\subsubsection{Work required for formation of the very small microscopic impression (A)}

' $A$ ' is calculated by Equation (8):

$$
F \cdot h_{\text {real }}^{\text {mean }}=A ; \quad[\mathrm{N}] \cdot[\mathrm{m}]=[\mathrm{J}]
$$

\subsubsection{Area of real impression $\left(\mathrm{S}_{\text {real }}\right)$}

$S_{\text {real }}$ is calculated from the experimentally obtained $d_{\text {real }}^{\text {mean }}$-values using Equation (9):

$$
\frac{2 \cdot 0.38 \cdot\left(d_{\text {real }}^{\text {mean }}\right)^{2}}{\sqrt{2}}=S_{\text {real }} ; \quad\left[\mathrm{m}^{2}\right]
$$

\subsubsection{Relation between $\sigma^{\mathrm{MHV}}, \mathrm{CRP}$ (a) and dreal}

Having in mind the steps already mentioned, $\sigma^{M H V}$ can be defined as the work, performed on a single unit area of real impression $\left(S_{\text {real }}\right)$ as given by Equation (10):

$$
\frac{A}{S_{\text {real }}}=\sigma^{M H V} ;\left[\frac{\mathrm{J}}{\mathrm{m}^{2}}\right]
$$

Summarizing Equations (4)-(9) a simple expression for $\sigma^{M H V}$ can be derived, as given by Equation (11):
$\sigma^{M H V}=a \cdot K \cdot \frac{d_{1 \mu \mathrm{m}}^{2}}{d_{\text {real }}^{\text {mean }}} ; K=\frac{1.8544}{7} ;\left[\frac{\mathrm{J}}{\mathrm{m}^{3}}\right] \cdot \frac{\left[\mathrm{m}^{2}\right]}{[\mathrm{m}]}=\left[\frac{\mathrm{J}}{\mathrm{m}^{2}}\right]$

Equation (11) represents a simple $\sigma^{M H V}-$ dependence on $d_{\text {real }}^{\text {mean }}$ and CRP $(a)$.

\subsection{Advantages and limitations of the new approach to the evaluation of $\sigma^{\mathrm{MHV}}$}

Another approach to calculation of surface free energy using microhardness data is described by Balta Calleja et al. [1, 5]. It is based on Equation (12):

$$
\frac{2 \sigma}{\Delta h}=l_{c} \cdot\left(\frac{H_{c}^{0}}{H_{c}}-1\right)
$$

where $\sigma$ is the surface free energy; $\Delta h$ - the energy required for plastic deformation of the crystals; $l_{c}$ - the average crystal thickness; $H_{c}^{0}$ - the microhardness of an infinitely thick crystal (maximum possible value of energy dissipated through plastic deformation); $H_{c}$ - the intrinsic microhardness of the crystalline phase.

Comparing Equations (11) and (12) we should to point out that:

- Equation (11) is a very simple expression of $\sigma^{M H V}$ dependence on the microhardness data. $\sigma^{M H V}$ depends on two parameters (CRP $(a)$ and $d_{\text {real }}^{\text {mean }}$ ) derived from a direct microhardness measurement. The surface free energy was calculated direct from the mean diagonal length of the impression $(d)$ using simple mathematical transformations.

- Despite of microhardness measurement, Equation (12) requires data from other methods:

- The average crystal thickness $\left(l_{c}\right)$ has to be determined by SAXS.

- Degree of crystallinity (determined by WAXS or DSC) is needed for calculating $H_{c}$.

- Expression (12) is applicable to semi-crystalline polymers like polyethylene samples of different structure [5, 6] and polymer blends [7]. We describe for the first time in this paper the application of Equation (11) to $\gamma$-irradiated PEUHMW. 


\section{Experimental}

\subsection{Materials}

PE-UHMW samples, prepared according to Bulgarian patent, Reg №30049 [8] (modified four component Ziegler-Natta catalytic system has been used), were supplied by Neftochim - Burgas. Molecular weight was determined by Vapor pressure osmometry $\left(M_{n}=1.28 \cdot 10^{5}\right)$ and by light scattering $\left(M_{w}=1.2 \cdot 10^{6}\right)$.

Test samples (4 $\mathrm{mm}$ thick) were prepared by pressing at $10-200 \mathrm{kN}$ and subsequent sintering at $160-200^{\circ} \mathrm{C}$.

\subsection{Methods}

\subsection{1. $\gamma$-irradiation}

The $\gamma$-irradiation was carried out by a $\gamma^{60} \mathrm{Co}$ source at room temperature in air at a dose rate of $10 \mathrm{kGy} / \mathrm{h}$. The samples were irradiated with 11 different doses: 1, 2, 5, 10, 20, 50, 100, 200, 500, 1000 and $1500 \mathrm{kGy}$. The doses were selected in such a way that their logarithmic values should increase linearly. In order to observe this dependence clearly and for convenience, the dose of the un-irradiated sample was selected to be $0.1 \mathrm{kGy}$. In this way the graphical presentation of the data can be easier understood and the dose dependences of the parameters calculated easier anticipated.

\subsubsection{Vickers microhardness (MHV)}

Microhardness was determined by the Vickers method. The experiment was carried out on a standard MHV tester mhp-160 for light microscope NU-2 (Germany). The loads applied were 0.0123 , $0.0245,0.049,0.098,0.196,0.392,0.785$ N. Similarly to the dose range, the loads were selected in such a way that their logarithmic values should increase linearly. This choice is based on the fact that MHV is a linearly logarithmic dependent quantity ([1], p. 56), eq. (3.2). In all experiments loading time was $30 \mathrm{~s}$.

\section{Results and discussion}

\subsection{Meyer's lines}

The Meyer's lines obtained are given on Figure 1. The dependence follows the Meyer's power law (Equation (2a)), which proves the reliability of the
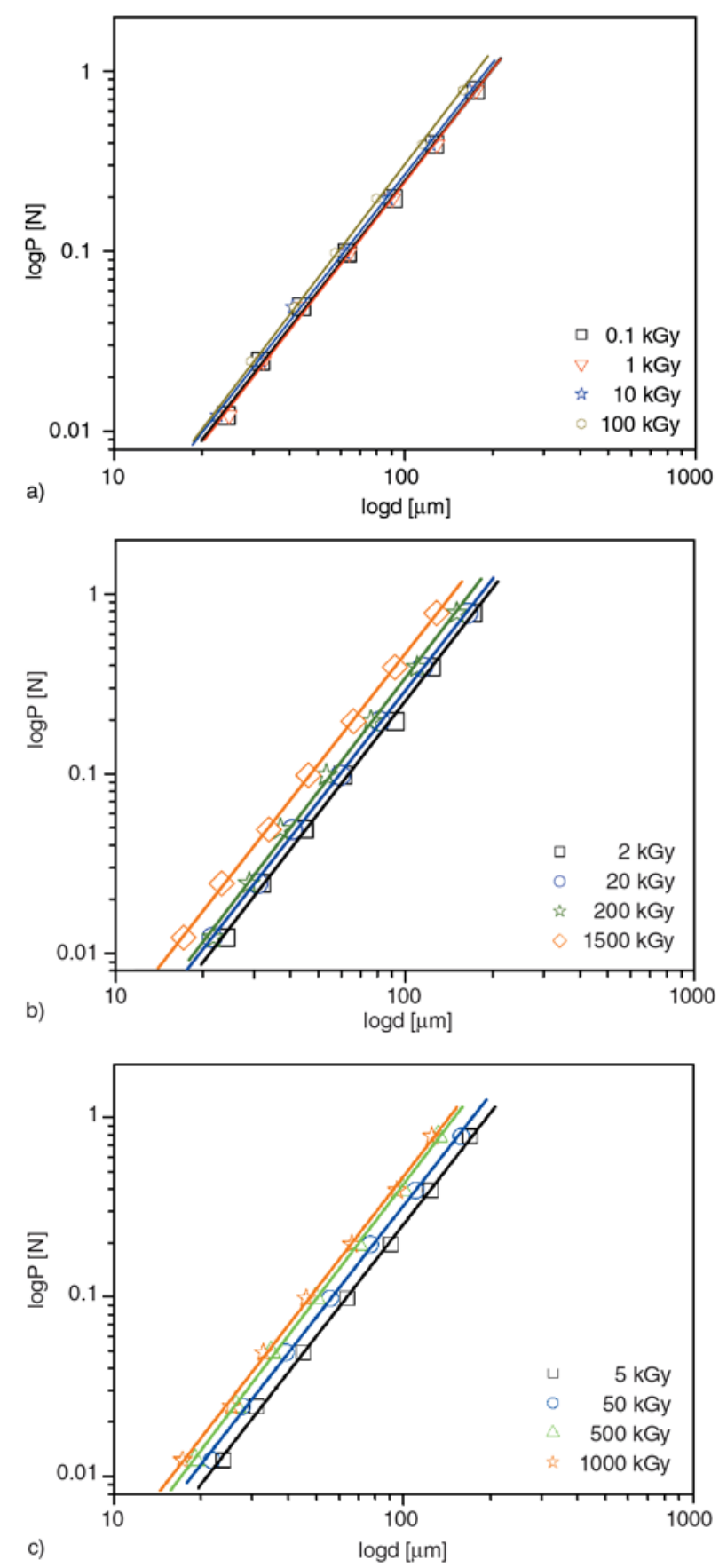

Figure 1. Meyer's lines for $\gamma$-irradiated PE-UHMW

obtained MHV data. According to the values of the correlation coefficient $(R)$ and standard deviation (SD), a good correlation between the load applied $(P)$ and the indentation diagonal length $(d)$ may be inferred from the experimental data (Table 1).

\subsection{Slope data ( $n$ values)}

As already mentioned the general requirement is $n=2$. The $n$-values (Table 2, Figure 2) of Meyer's lines for the PE-UHMW samples studied vary in the range $2.05-2.1$. The mean slope-value $\left(n_{\text {mean }}\right)$ is 
2.07. 'nmean' is very close to the slope-value, required for applying Equation (11).

Table 1. Correlation coefficient $(R)$ and standard deviation (SD) of the Meyer's lines

\begin{tabular}{|c|c|c|}
\hline Dose [kGy] & R & SD \\
\hline 0.1 & 0.9993 & 0.03 \\
\hline 1 & 0.9993 & 0.03 \\
\hline 2 & 0.9992 & 0.03 \\
\hline 5 & 0.9995 & 0.02 \\
\hline 10 & 0.9992 & 0.03 \\
\hline 20 & 0.9996 & 0.02 \\
\hline 50 & 0.9989 & 0.03 \\
\hline 100 & 0.9994 & 0.02 \\
\hline 200 & 0.9983 & 0.04 \\
\hline 500 & 0.9995 & 0.02 \\
\hline 1000 & 0.9991 & 0.03 \\
\hline 1500 & 0.9998 & 0.01 \\
\hline
\end{tabular}

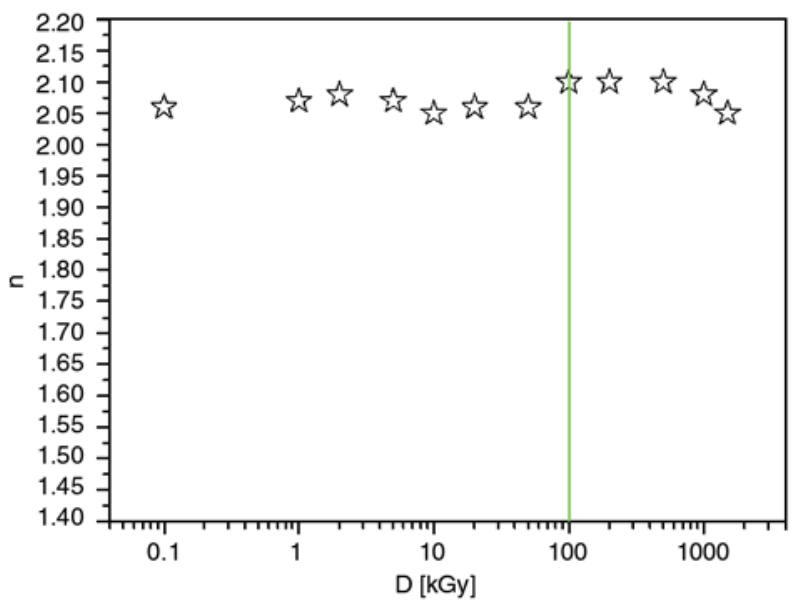

Figure 2. Dependence of the Meyer's lines slope value ( $n$ ) on the dose $(D)$; the symbol for dose is $D$, [kGy], in contrast to the mean diagonal length $d$, $[\mu \mathrm{m}]$. Do not confuse the two parameters, please.

\subsection{Surface free energy $\left(\sigma^{\mathrm{MHV}}\right)$ data and their reliability}

All results are summarized in Table 2. Experimental values for surface free energy (Figure 4, $\sigma^{M H V}=$ $0.057-0.173 \mathrm{~J} / \mathrm{m}^{2}$ ) show good agreement with literature data [5, 9, 10]. Balta Calleja et al. [5] reported $\sigma=0.079-0.091 \mathrm{~J} / \mathrm{m}^{2}$ for a series of PE samples of different molecular weights. Non-isothermal kinetics investigations [9] show similar results: $\sigma^{m, c}=$ $0.040-0.085 \mathrm{~J} / \mathrm{m}^{2}$. According to Wunderlich [10] $\sigma=0.06 \mathrm{~J} / \mathrm{m}^{2}$.

\subsection{Radiation effects}

For all samples investigated $\log a$ (Figure 3), CRP (Figure 4) and $\sigma^{M H V}$ (Figure 5) exhibit well defined dose $(D)$ dependence. It corresponds to the radiation effects theory for PE-UHMW [9] and ultra high molecular weight polyethylene oxide

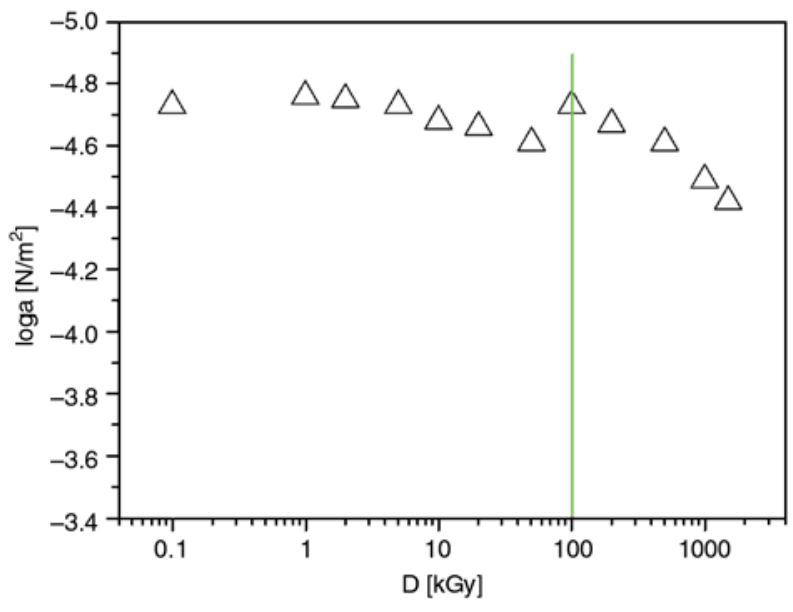

Figure 3. Dependence of the Meyer's lines cut values $(\log a)$ on the dose $(D)$

Table 2. Microhardness data for PE-UHMW samples $\gamma$-irradiated with different doses

\begin{tabular}{|c|c|c|c|c|c|}
\hline D [kGy] & $\mathbf{n}$ & $\log a\left[N / m^{2}\right]$ & $a \cdot 10^{-5}\left[\mathrm{~N} / \mathrm{m}^{2}\right]$ & $\mathrm{d}_{\mathrm{real}}^{\text {mean }} \cdot \mathbf{1 0}^{-5}[\mathrm{~m}]$ & $\sigma^{\mathrm{MHV}}\left[\mathrm{J} / \mathbf{m}^{2}\right]$ \\
\hline 0.1 & 2.06 & -4.73 & 1.86 & 7.96 & 0.062 \\
\hline 1 & 2.07 & -4.76 & 1.74 & 8.02 & 0.057 \\
\hline 2 & 2.08 & -4.75 & 1.78 & 7.82 & 0.060 \\
\hline 5 & 2.07 & -4.73 & 1.86 & 7.83 & 0.063 \\
\hline 10 & 2.05 & -4.68 & 2.09 & 7.64 & 0.072 \\
\hline 20 & 2.06 & -4.66 & 2.19 & 7.33 & 0.079 \\
\hline 50 & 2.06 & -4.61 & 2.45 & 6.99 & 0.093 \\
\hline 100 & 2.10 & -4.73 & 1.86 & 7.21 & 0.068 \\
\hline 200 & 2.10 & -4.67 & 2.14 & 6.82 & 0.083 \\
\hline 500 & 2.10 & -4.61 & 2.45 & 6.15 & 0.110 \\
\hline 1000 & 2.08 & -4.49 & 3.24 & 5.82 & 0.150 \\
\hline 1500 & 2.05 & -4.42 & 3.80 & 5.81 & 0.170 \\
\hline
\end{tabular}




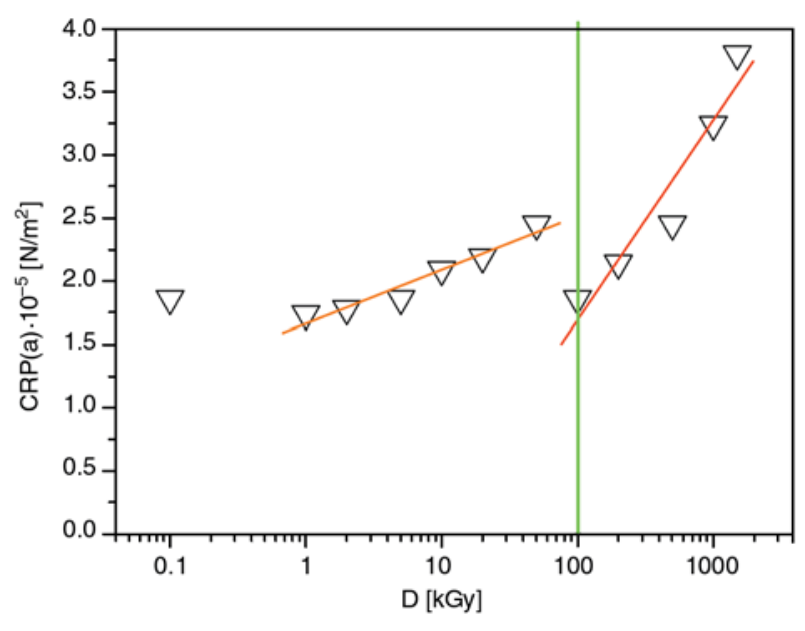

Figure 4. Dose dependence of CRP (a) values

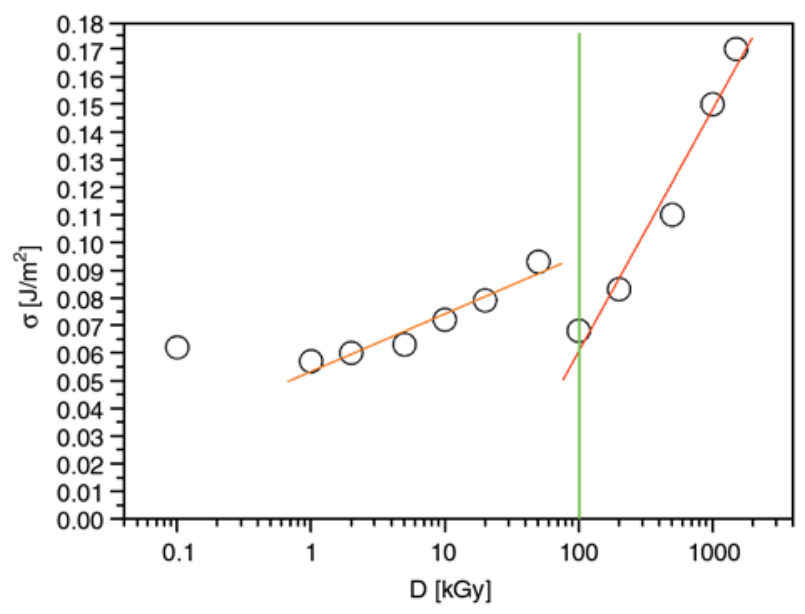

Figure 5. Dose dependence of $\sigma^{M H V}$ values

(UHMWPEO) [11, 12]. Critical dose is observed at $100 \mathrm{kGy}$ - the borderline between two radiation effects: radiation annealing and radiation melting. The surface free energy $\left(\sigma^{M H V}\right)$ shows a good linear dependence on the dose for each (of the) radiation effect. The first $\sigma^{M H V}$ value (for the un-irradiated sample, $D=0.1 \mathrm{kGy}$, Figure 5) is used as a referent value. Two dose ranges were investigated and two radiation effects were observed:

- $D=1-50 \mathrm{kGy}(D<100 \mathrm{kGy})$ : the effect of radiation annealing;

- $D=100-1500 \mathrm{kGy}(D \geq 100 \mathrm{kGy})$ : the effect of radiation melting.

The radiation effects result in changes in polymer structure. These changes proceed in the inter-crystalline (amorphous) lamellar spaces (Figure 6).

Radiation annealing and radiation melting are similar to the temperature annealing and temperature melting but the variable is the dose, not the temperature.

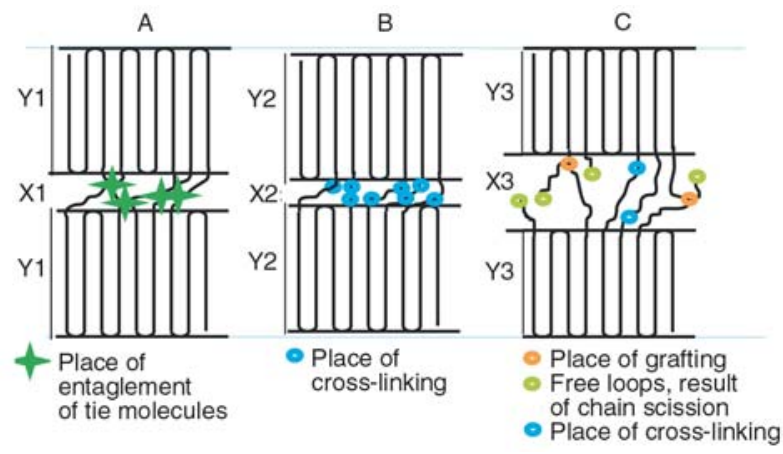

Figure 6. Lamellar structure of the polymer caused by $\gamma$-irradiation (B and C) compared to that of the un-irradiated sample (A): B - irradiated sample during radiation annealing; $\mathrm{C}$ - irradiated sample during radiation melting. $Y_{1}, Y_{2}, Y_{3}-$ lamellar crystal part: $Y_{1}=Y_{2}>Y_{3} ; X_{1}, X_{2}, X_{3}-$ lamellar amorphous part: $X_{1}>X_{2}<X_{3}$

In the case of PE-UHMW the $\gamma$-irradiation causes simultaneous chain scission and recombination in the lamellar crystal parts. According to chain mechanism, chain scission and chain cross-linking proceed simultaneously in the amorphous lamellar part as well.

For these two processes, it is supposed that the macromolecules absorb energy, hence radiation defects/excitons occur.

In the range of radiation annealing ( $D<100 \mathrm{kGy})$, excitons move along the macromolecular segments in the lamellar crystal part and are pushed out to the lamellar surface. This results in decrease of crystal defects and in increase of total crystallinity. The effect observed is denoted as radiation annealing, similar to temperature annealing. Low numbers of radiation defects/excitons are caused by irradiation doses in the range $D=1-100 \mathrm{kGy}$. They decrease the inter-lamellar stress due to chain-scission of the entangled and stressed tie molecules of PEUHMW. Hence at very low doses (Figure 5, points at $D=1 \mathrm{kGy}$ and $D=2 \mathrm{kGy}$ ) the lamellar surface free energy $\left(\sigma^{M H V}\right)$ is lower compared to that of the un-irradiated sample ( $D=0.1 \mathrm{kGy})$. At doses, close to the critical value ( $D=100 \mathrm{kGy})$, the number of the excitons/radiation defects on the lamellar surface slightly increases. The defects cause mainly cross-linking. It slightly progresses to the surface and becomes more stressed, causing slow increase in the surface free energy $\left(\sigma^{M H V}\right)$ with the increasing doses (Figure 5, orange line).

In the range of radiation melting ( $D \geq 100 \mathrm{kGy})$ much more excitons are formed. The number of 
high energy excitons increases, so that the chain scission and cross-linking proceed only at the crystal-amorphous boundary. In this dose range the amorphous part thickness increases at the expense of the crystal one. The process is similar to the surface melting and therefore is denoted as radiation melting [11]. The increase of the dose (starting at $D=100 \mathrm{kGy}$ ) results in increase of the lamellar surface and the defects expand very quickly. Surface free energy $\left(\sigma^{M H V}\right)$ increases rapidly (Figure 5, red line).

\section{Conclusions}

Using direct Vickers microhardness data $(d)$ a new approach to calculating of surface free energy has been developed. After simple mathematical transformations, the parameters CRP $(a)$ and $d_{\text {real }}^{\text {mean }}$ were calculated and an equation for $\sigma^{M H V}$ was deduced. This equation is applicable only at the general requirement $(n=2)$ - i.e Meyer's lines slope data (n) has to fulfill this requirement. The surface free energy $\left(\sigma^{M H V}\right)$ data, obtained by applying our new approach to estimation of surface free energy based on Vickers microhardness data, show very good agreement with the literature $[5,9,10]$. It has been shown, that our new approach has rendered reliable results for the PE-UHMW samples studied. The $\sigma^{M H V}$ values obtained are dose dependent and correspond to the radiation effects theory.

\section{Acknowledgements}

Maya Staneva would like to thank the Structural Funds and Educational Programs Directorate for the financial support within the frame of the Project 'Support for the development and realization of $\mathrm{PhD}$-students, post-docs and young researchers in the field of polymer chemistry, physics and engineering', Grant № BG051PO001/07/3.3-02/51 51.

\section{References}

[1] Balta Calleja F. J., Fakirov S.: Microhardness of polymers. Cambridge University Press, Cambridge (2000).

[2] Zamfirova G., Dimitrova A.: Some methodological contributions to the Vickers microhardness technique. Polymer Testing, 19, 533-542 (2000). DOI: $10.1016 / \mathrm{S} 0142-9418(99) 00023-9$
[3] Zamfirova G., Lorenzo V., Benavente R., Pereña J. M.; On the relationship between modulus of elasticity and microhardness. Journal of Applied Polymer Science, 88, 1794-1798 (2003).

DOI: $10.1002 / a p p .11788$

[4] Zamfirova G., Pereña J. M., Benavente R., Perez E., Cerrada M. L., Nedkov E.: Mechanical properties of ultra high molecular weight polyethylene obtained with different cocatalyst systems. Polymer Journal, 34, 125-131 (2002).

DOI: $10.1295 /$ polymj.34.125

[5] Baltá Calleja F. J., Santa Cruz C., Bayer R. K., Kilian H. G.: Microhardness and surface free energy in linear polyethylene: The role of entanglements. Colloid and Polymer Science, 268, 440-446 (1990). DOI: $10.1007 / \mathrm{BF} 01411002$

[6] Flores A., Baltá Calleja F. J., Bassett D. C.: Microhardness studies of chain-extended PE: I. Correlations to microstructure. Journal of Polymer Science Part B: Polymer Physics, 37, 3151-3158 (1999). DOI: 10.1002/(SICI)1099-0488(19991101)37:

\section{1<3151::AID-POLB24>3.0.CO;2-E}

[7] Flores A., Aurrekoetxea J., Gensler R., Kausch H. H., Baltá Calleja F. J.: Microhardness-structure correlation of iPP/EPR blends: Influence of molecular weight and EPR particle content. Colloid and Polymer Science, 276, 786-793 (1998). DOI: $10.1007 / \mathrm{s} 003960050311$

[8] Glavchev I., Radenkov Ph., Kabivanov V., Kircheva R., Yalamova T.: Method for preparation of PEUHMW. Bulgarian patent. Reg. № 30049, Bulgaria (1975).

[9] Staneva M., Nedkov E.: Kinetic investigation of $\gamma$ irradiated PE-UHMW over non-isothermal processes according to renewed Nedkov-Atanasov approach. Radiation effects, in 'Reactor powder morphology' (eds.: Myasnikova L., Lemstra P. J.) Nova Science Publishers Inc., New York, in press (2009).

[10] Wunderlich B.: Macromolecular Physics, vol. 2. Mir, Moskva (1979).

[11] Nedkov E., Tsvetkova S.: Effect of gamma irradiation on the melting characteristics of ultra high molecular weight poly (ethylene oxide). Radiation Physics and Chemistry, 44, 251-256 (1994). DOI: $10.1016 / 0969-806 \mathrm{X}(94) 90002-7$

[12] Nedkov E., Tsvetkova S.: Effect of gamma irradiation on the crystalline structure of ultra high molecular weight poly (ethylene oxide). Radiation Physics and Chemistry, 43, 397-401 (1994). DOI: $10.1016 / 0969-806 X(94) 90034-5$ 